\title{
FOUR DE LABORATOIRE POUR FUSION DE MÉTAUX ET ALLIAGES « PROPRES »
}

\author{
O. BETHOUX, B. CORNUT et M. FERRARI \\ C. N. R. S., Centre de Recherches sur les Très Basses Températures \\ Cédex 166, 38, Grenoble-Gare \\ (Reçu le 21 août 1970)
}

\begin{abstract}
Résumé. - Un four de laboratoire destiné à la fusion et la coulée en lingotière refroidie, de petits échantillons de métaux et alliages, est décrit. Mettant à profit à la fois la fusion sur sole refroidie et la lévitation électromagnétique, il permet dans sa version actuelle, de fondre et allier dans de bonnes conditions, de pureté et d'homogénéité tous les métaux dont le point de fusion est inférieur à $3000^{\circ} \mathrm{C}$.
\end{abstract}

\begin{abstract}
A laboratory furnace for melting and then casting in a cooled mold, small samples of metals and alloys, is described. Using both melting on a cooled base-plate and levitation, it can be used for melting and alloying with good purity and homogeneity, all metals whose melting point is less than $3000^{\circ} \mathrm{C}$.
\end{abstract}

Introduction. - Malgré la disponibilité croissante sur le marché de métaux très purs, la préparation d'échantillons d'alliages métalliques «propres », dans lesquels les impuretés autres que le ou les solutés volontairement introduits sont présentes en quantité négligeable, continue de poser en général de sérieux problèmes aux laboratoires et en particulier à ceux de physique du solide. En effet, compte tenu de la diversité des métaux et alliages souvent étudiés par un même laboratoire, il est nécessaire de pouvoir disposer d'une méthode de préparation aussi universelle que possible, susceptible de fournir des échantillons à la fois «propres" et homogènes. De plus, en raison du prix très élevé des métaux très purs, la méthode doit pouvoir s'appliquer, avec un minimum de pertes, à de faibles quantités de métal, la forme, la masse et la concentration des lingots obtenus étant les plus proches possibles de celles des échantillons désirés.

Les méthodes classiques de fusion, associées à divers types de chauffage, ne répondent pas, en général, à l'ensemble de ces conditions et c'est afin de les satisfaire au mieux que nous avons conçu et réalisé un petit four de laboratoire permettant de fondre et de couler des échantillons d'un volume de l'ordre $\mathrm{du}^{3} \mathrm{~cm}^{3}$, dont le principe de fonctionnement réalise un compromis entre la fusion en lévitation électromagnétique [1] et la fusion sur sole refroidie suivie d'une coulée en lingotière froide.

Principe de fonctionnement et réalisation. - Un schéma de principe est représenté sur la figure 1. Le métal à fondre $(a)$ repose sur la partie centrale en forme d'entonnoir d'une sole $(b)$ parcourue par une

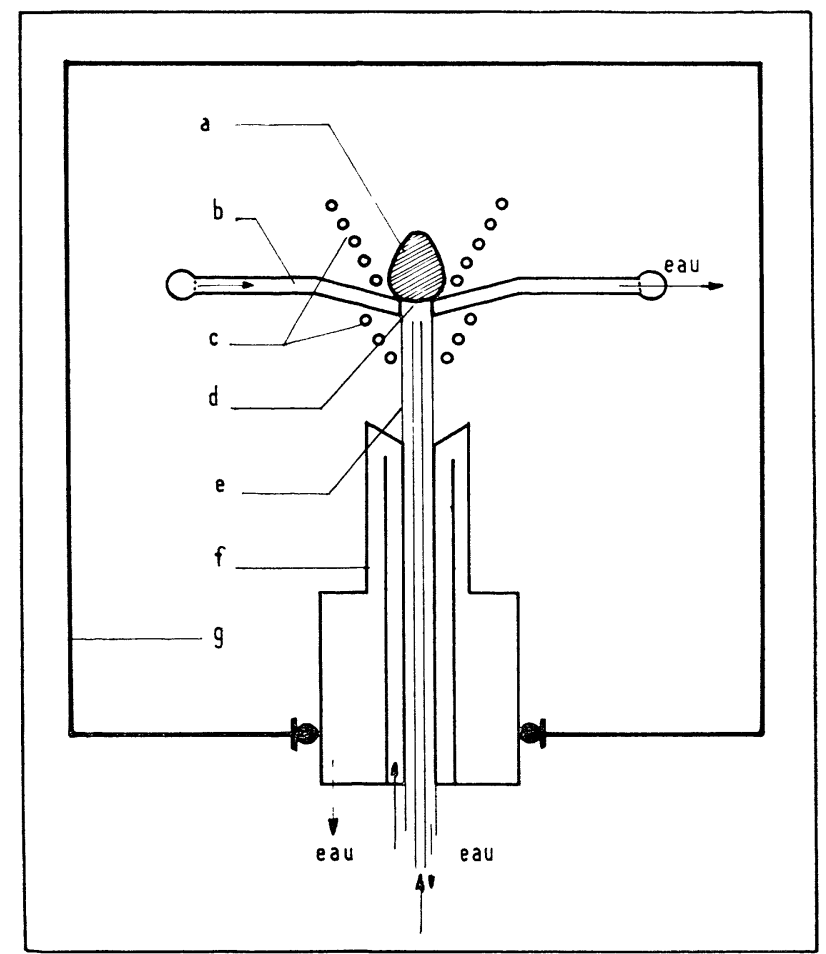

Fig. 1. - Schéma de principe du four : $a$ ) métal fondu; $b$ ) sole refroidie ; $c$ ) inducteur ; $d$ ) trou de coulée ; $e$ ) piston refroidi ; $f$ ) lingotière refroidie ; $g$ ) enceinte étanche.

circulation d'eau ; il est chauffé grâce à un inducteur (c) refroidi également à l'eau et relié à un générateur haute fréquence fonctionnant à $400 \mathrm{kHz}$. Un trou de coulée $(d)$ ménagé dans la partie centrale de la sole et situé dans l'axe de l'inducteur, est obturé, pendant 
la fusion du métal, par la partie supérieure d'un piston $(e)$, refroidi à l'eau, coulissant dans une lingotière $(f)$ également refroidie à l'eau. L'ensemble du dispositif est monté dans une enceinte transparente étanche $(g)$ permettant de travailler sous vide $\left(10^{-6}\right.$ torr $)$ ou sous atmosphère contrôlée. La sole, l'inducteur, le piston et la lingotière sont réalisés en cuivre. Sous l'action des forces électromagnétiques qui tendent à le soulever, le métal une fois fondu ne s'étale pas sur la sole mais se rassemble et prend une forme de "poire » caractéristique. Cet effet, en réduisant la surface de contact entre le métal et la sole refroidie, limite les pertes thermiques et permet d'obtenir la fusion de la totalité du métal en un bain unique. La coulée est réalisée en abaissant brusquement le piston dans la lingotière et en interrompant simultanément le courant haute fréquence dans l'inducteur.

Afin de limiter les pertes de puissance, la sole est constituée de tubes de cuivre parallèles non jointifs de $4 \mathrm{~mm}$ de diamètre extérieur et de $0,5 \mathrm{~mm}$ d'épaisseur. Pour pouvoir couler le maximum de métal liquide, il est nécessaire que le profil de la partie centrale de la sole entourant le trou de coulée, soit le plus régulier possible. Pour obtenir une telle régularité de profil en conservant la structure tubulaire de la sole, un dépôt électrolytique de cuivre a été réalisé sur les tubes préalablement mis en forme et assemblés puis la partie centrale conique a été usinée dans le dépôt de cuivre (Fig. 2 et 3) avec un angle au sommet de 130 . L'espacement des tubes de cuivre a été conservé grâce à des cales d'épaisseur en aluminium, mises en place avant le dépôt électrolytique et retirées après usinage.

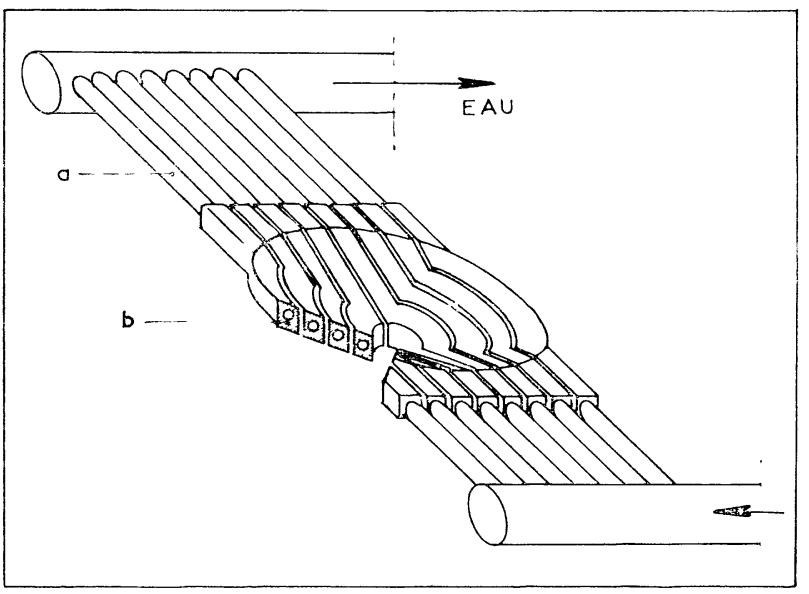

FIG. 2. - Détail de la sole refroidie : $a$ ) tubes de cuivre diamètre $4 \mathrm{~mm}$, épaisseur 0,$5 ; b$ ) cuivre déposé électrolytiquement.

Nous ne sommes pas parvenus jusqu'ici à mettre au point un inducteur universel et plusieurs formes d'inducteur (Fig. 3) ont été nécessaires pour fondre les différents métaux sur lesquels ont porté les essais. Notons à ce sujet, que les meilleures formes d'inducteur pour la lévitation électromagnétique [2] ne le sont pas nécessairement ici. Une vue générale du four ouvert est donnée sur la figure 4 où l'on pourra remarquer

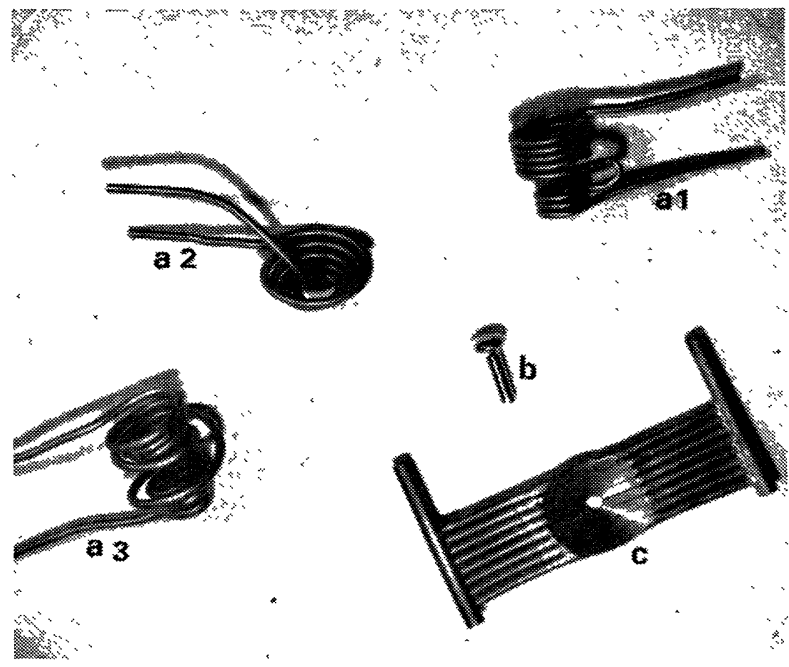

FIG. 3. - $a$ ) Formes d'inducteur utilisées :

$a_{1}$ ) inducteur pour $\mathrm{Au}, \mathrm{Pd}, \mathrm{Fe}, \mathrm{Zr}, \mathrm{V}, \mathrm{Nb}$ $a_{2}$ ) inducteur pour $\mathrm{Ag}, \mathrm{Au}, \mathrm{Cu}, \mathrm{Fe}, \mathrm{Pt}$ $a_{3}$ ) inducteur pour $\mathrm{Au}, \mathrm{Cu}, \mathrm{Pt}, \mathrm{Nb}$

b) échantillon d'or

c) sole refroidie

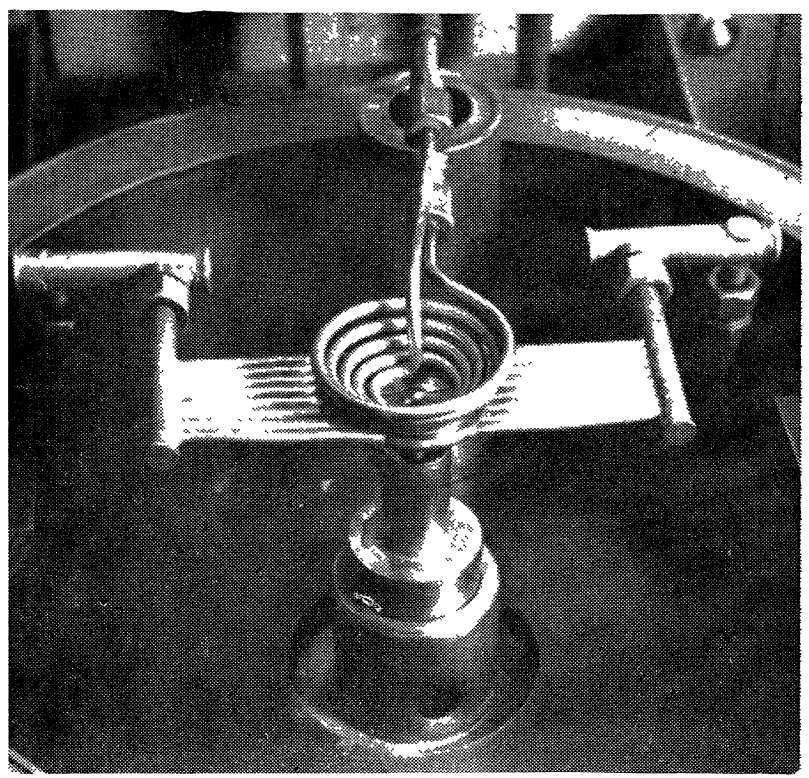

FIG. 4. - Vue d'ensemble du four ouvert.

l'extrême simplicité du dispositif, aucune pièce n'étant susceptible de chauffer et par conséquent de dégazer, pendant la fusion sous vide.

Résultats obtenus. - Les essais de fusion et de coulée ont porté sur une gamme étendue de métaux possédant des points de fusion, des densités, des tensions superficielles et des conductivités électrique et thermique très différentes. Avec le générateur haute fréquence STEL de $17 \mathrm{~kW}$ dont nous disposions, le plus haut point de fusion atteint a été celui du tantale, qu'il a été possible de fondre mais non de couler. Par contre, $\mathrm{Ag}, \mathrm{Au}, \mathrm{Cu}, \mathrm{Fe}, \mathrm{Pd}, \mathrm{Pt}, \mathrm{Zr}, \mathrm{V}, \mathrm{Nb}$ ont été 
fondus en quelques minutes et coulés en lingots de $7 \mathrm{~mm}$ de diamètre et 20 à $25 \mathrm{~mm}$ de long (Fig. 3). Nous avons pu remarquer que pour obtenir une coulée presque totale du métal liquide, celui-ci devait être bien centré au-dessus du trou de coulée, ce qui nécessite une forme et un positionnement corrects de l'inducteur.

Contamination. - L'une des premières applications projetées pour ce four étant la préparation d'alliages très dilués en vue d'étude de magnétisme aux très basses températures, nous nous sommes tout particulièrement intéressés à la contamination des échantillons par les impuretés magnétiques. L'aimantation à saturation $\sigma_{\mathrm{s}}$, des impuretés magnétiques étant fonction de leur concentration dans un métal non magnétique, nous nous sommes servis des mesures d'aimantation à très basses températures pour contrôler la contamination. Les essais ont été effectués avec du platine JohnsonMatthey contenant moins de 10 p.p.m. d'impuretés au total. D'après l'analyse fournie par le fabricant, le platine contient 2 p. p. m. de fer, les autres impuretés magnétiques n'étant pas détectables par spectroscopie d'émission. Le tableau I donne les valeurs de $\sigma_{\mathrm{s}}$ obtenues pour divers échantillons à $1,2^{\circ} \mathrm{K}$. L'échantillon 0 est du platine, mesuré tel que nous l'avons reçu sous forme de fil de $1 \mathrm{~mm}$ de diamètre; les échantillons 1 à 3 ont été fondus et coulés avec le four décrit ci-dessus ; l'échantillon 4 a été fondu sous vide en creuset d'alumine (pureté 99,9\%) pendant une heure à $1850^{\circ} \mathrm{C}$, à l'aide d'un four à résistor de tungstène.

Sur le tableau I ont été également portées les concentrations de fer, $C_{\mathrm{Fe}}$, correspondant aux valeurs de $\sigma_{\mathrm{s}}$ en supposant que toutes les impuretés magnétiques sont du fer ainsi que la contamination apportée par la fusion, $\Delta \sigma_{\mathrm{s}}$ et $\Delta C_{\mathrm{Fe}}$. Le moment magnétique associé à un atome de fer dans le platine a été pris égal à 6,46 magnétons de Bohr [3] [4].

\section{TABLEAU I}

\begin{tabular}{|c|c|c|c|c|c|}
\hline No échantillon (*) & 0 & 1 & 2 & 3 & 4 \\
\hline$\sigma_{\mathrm{s}} 10^{-4}$ u. é. m./g & 31 & 35 & 35 & 43 & 391 \\
\hline$C_{\mathrm{Fe}}$ p.p.m. $(*)$ & 4,8 & 5,4 & 5,4 & 6,7 & 60 \\
\hline$\Delta \sigma_{\mathrm{s}} 10^{-4}$ u. é. m. $/ \mathrm{g}$ & 0 & 4 & 4 & 12 & 360 \\
\hline$\Delta C_{\mathrm{Fe}}$ p. p. m. $\left(^{*}\right)$ & 0 & 0,6 & 0,6 & 1,9 & 55 \\
\hline
\end{tabular}

$\left(^{*}\right)$ Voir le texte.

On peut voir sur ce tableau que la contamination en impuretés magnétiques au cours de la fusion à l'aide du four décrit, est dans tous les cas inférieure au contenu initial d'impuretés du métal, et qu'un gain considérable a été obtenu par rapport à la fusion en creuset d'alumine.
Etant donné la méthode de fusion utilisée ici, le cuivre est le contaminant le plus probable ;

$$
9 \text { p. p. m. } \pm 3
$$

de cuivre ont été trouvés dans l'échantillon $n^{\circ} 3$. L'analyse a été faite par spectrographie d'absorption atomique après séparation du platine sur résine échangeuse d'ions. Bien que la quantité de cuivre introduite au cours de la fusion ne soit pas négligeable par rapport au contenu initial d'impuretés du métal, nous n'avons pas cherché, dans une première étape, à réduire la contamination par le cuivre, car celui-ci n'est pas gênant pour les mesures projetées sur les alliages fabriqués à l'heure actuelle avec ce four.

Homogénéité. - Nous avons testé l'homogénéité macroscopique des lingots, d'une part, en mesurant séparément l'aimantation de deux moitiés d'un lingot d'alliage Pt-Co 0,56 at. \%, et d'autre part, en analysant un lingot d'alliage $\mathrm{Cu}-\mathrm{Fe} 0,02$ at. $\%$ ainsi que le résidu non coulé, demeuré sur la sole du four. Dans les deux cas, aucune différence n'a été observée entre les fractions, à la reproductibilité des mesures près, soit $10^{-3}$ pour les mesures d'aimantation et quelque $10^{-2}$ pour les analyses.

Conclusion. - La méthode de fusion présentée ici, et le four qui la met en œuvre, particulièrement simple à réaliser, possèdent un certain nombre d'avantages :

- bain métallique unique assurant l'homogénéité des alliages et l'utilisation complète des métaux constitutifs ;

- absence de creuset réfractaire éliminant toute pollution du métal par le réfractaire lui-même ou les impuretés qu'il contient;

- mode de chauffage permettant la fusion sous vide ou atmosphère contrôlée, jusqu'à des températures de $3000^{\circ} \mathrm{C}$, tout en assurant un brassage énergique du métal liquide autorisant des temps de fusion très courts ;

- possibilité de coulée en lingotière refroidie pour contrôler la forme des échantillons et assurer un refroidissement rapide du métal;

- possibilité d'opérer sur des volumes de métal de l'ordre $\mathrm{du} \mathrm{cm}^{3}$.

L'ensemble de ces avantages permet, avec un appareillage unique et relativement peu onéreux, de fondre et allier la presque totalité des métaux, dans de bonnes conditions de "propreté ", d'homogénéité et de rapidité. Ce type de four s'avère donc bien adapté à la préparation d'échantillons métalliques pour le laboratoire de métallurgie ou de physique du solide.

\section{Bibliographie}

[1] Okress (E. C.) et al., J. of App. Phys., 1952, 23, 545. [3] Crangle (J.) and Scott (W. R.), J. of App. Phys.,

[2] Fromm (E.) und Jehn (H.), Z. Metallkde, 1967. $1965,36,921$.

[4] Maley (M. P.), Taylor (R. D.) and Thompson (J. L.), J. of App. Phys., 1967, 38, 1249. 